Aims The aim of our study was to assess the existing infection control guidelines and their enforcement for visitors attending tertiary level NICUs across England and to recommend a standard set of guidelines to reduce the risk of infection in infants on NICU.

Methods Our study included 46 level 3 NICUs across England during November 2013-March 2014. A survey questionnaire was set and the sister in charge was contacted by telephone.

Results Among 46 units, 46(100\%) responded to the survey. 27 $(58.7 \%)$ provided infection control leaflets for visitors, all of those included hand washing. 15 of the $27(55.5 \%)$ had multilingual leaflets. $34(73.9 \%)$ used a cot-side nurse or receptionist to enforce infection control policies. 11(23.9\%) had no restricted visiting times, only if accompanied by parents. 42(91.3\%) allowed siblings, however 2 excluded winter, 1 disallowed siblings under 6, and 1 only in infectious outbreaks. 26 (56.5\%) had a limited handling policy. All allowed kangaroo care, 28 $(60.9 \%)$ of which stipulated only when the baby was stable. 10 $(21.7 \%)$ allowed it any time. $1(2.2 \%)$ only permitted on extubation and if lines were taken out. 42(91.3\%) prohibited wearing coats. $3(6.5 \%)$ had a bare below the elbow policy and 25 $(54.3 \%)$ asked that jewellery was removed. None of the units asked visitors to remove plain bands. All stipulated hand washing, however 44(95.6\%) stipulated hand gelling. 3(6.5\%) stipulated the wearing of gowns, and 4 (8.6\%) asked visitors to wear gloves, both of which were only necessary if the baby had an infection.

Conclusion Our study results have thrown light on variations in infection control policies for visitors to NICUs across England. A set of infection control guidelines and methods for their enforcement are proposed.

\section{G125(P) UK TRANSFUSION-ASSOCIATED NECROTISING ENTEROCOLITIS CASES IDENTIFIED THROUGH A MULTICENTRE AUDIT}

${ }^{1} \mathrm{~S}$ Hamad, ${ }^{2} \mathrm{~K}$ Jones, ${ }^{3} \mathrm{~K}$ Sim, ${ }^{4} \mathrm{~S}$ Cherian, ${ }^{5} \mathrm{~A}$ James, ${ }^{2} \mathrm{~S}$ Godambe, ${ }^{6} \mathrm{H}$ New, ${ }^{3} \mathrm{SS}$ Kroll, ${ }^{1} \mathrm{P}$ Clarke. ${ }^{1}$ Neonatal Unit, Norfolk and Norwich University Hospitals NHS Foundation Trust, Norwich, UK; ${ }^{2}$ Neonatal Unit, Imperial College Healthcare NHS Trust, London, UK; ${ }^{3}$ Department of Medicine, Section of Paediatrics, Imperial College, London, UK; ${ }^{4}$ Neonatal Unit, University Hospital of Wales, Cardiff, UK; ${ }^{5}$ Neonatal Unit, Royal Gwent Hospital, Newport, UK; ${ }^{6}$ Department of Paediatrics, Imperial College Healthcare NHS Trust/NHS Blood and Transplant, London, UK

\subsection{6/archdischild-2015-308599.124}

Aim Transfusion-associated necrotising enterocolitis (TANEC) has been reported from The Americas and various European countries at rates comprising $27-38 \%$ of necrotising enterocolitis (NEC) cases. While the role of packed red blood cell (PRBC) transfusion in causation of NEC remains debated, there are surprisingly few reports of TANEC in the UK setting. Our aim was to analyse the incidence of NEC and TANEC in UK NICUs using uniform definitions.

Methods We undertook a retrospective multicentre audit covering the period October 2011 to November 2014 in four tertiary-level UK NICUs. We assessed whether definite NEC cases (NEC diagnosed surgically via laparotomy, post-mortem, and/or a strict clinical-radiological diagnosis) were also TANEC cases (first onset of NEC symptoms within $48 \mathrm{~h}$ of commencement of a PRBC transfusion). Of participating NICUs, three introduced routine probiotic prophylaxis during the study period and one practised routine cessation of enteral feeding during PRBC transfusions.
Results 1608 (20.1\%) of 8007 babies admitted in the 38-month study period were very low birth weight (VLBW) and 68 (4.2\%) had definite NEC. Of these $15(22.1 \%)$ were TANEC; 34 (50.0\%) had received prior PRBC transfusion but were not TANEC; 19 (27.9\%) had received no prior PRBC transfusion. Across NICUs, the incidence of definite NEC ranged from 4.5-9.7 cases/year (3.6-7.8 per 100 VLWB admissions) and that of TANEC ranged from $0.50-1.95$ cases/year $(0.4-1.7$ per 100 VLWB admissions). The proportion of TANEC/NEC cases within individual NICUs ranged from 11\%-40\%. TANEC cases were of median birth weight $695 \mathrm{~g}$ (range: 527-1070 g) and birth gestation $25^{+1}$ weeks (range: $23^{+2}-27^{+0}$ weeks). Median age at start of the index PRBC transfusion was 18 days (range: 0-69 days). Overall, $12(80 \%)$ required surgical intervention and $9(60 \%)$ survived to discharge. Four (27\%) TANEC cases occurred in babies who had received prior probiotics.

Conclusion TANEC occurs in the UK in proximal association with PRBC transfusion at rates similar to those reported from other countries. Rates of NEC and TANEC vary widely between UK centres. A large prospective UK surveillance study is now indicated to improve the understanding of the causation of TANEC.

\section{G126(P) AN AUDIT ON HOME NASOGASTRIC TUBE FEEDING PRACTICES IN A REGIONAL NEONATAL UNIT}

${ }^{1} \mathrm{~N}$ Athiraman, ${ }^{2} \mathrm{R}$ Coombs, ${ }^{2} \mathrm{~J}$ Paulucy, ${ }^{2} \mathrm{D}$ Crossley, ${ }^{2} \mathrm{~J}$ Shaw. ${ }^{1}$ The Newcastle Upon Tyne Hospitals NHS Foundation Trust, Newcastle-Upon-Tyne, UK; ${ }^{2}$ Sheffield Teaching Hospitals NHS Foundation Trust, Sheffield, UK

\subsection{6/archdischild-2015-308599.125}

Introduction Infants who are borderline premature, low birthweight and multiple births have extended stay within the neonatal unit, primarily to establish feeds. Discharging these infants' home on nasogastric feeds (NGF) to establish feeds, would have significant benefits for family bonding and freeing neonatal cot spaces. However, there is a need for risk assessment as well as parental training. Aim To identify the benefits and safety of nasogastric feeding at home.

The objective is to identify the number of saved bed days and costs, infants' growth and support required at home

Methods This audit was conducted over a period of 18 months from January 2011 to June 2012. All the infants discharged home on NGF were identified from the community database and data collected from medical as well as community nursing notes. Two days were added to the total, as the unit protocol is to observe infants for $48 \mathrm{hrs}$ after fully established feeds, before discharging home.

Abstract G126(P) Table 1 Home NG feeding after early discharge, and their outcomes

\begin{tabular}{ll}
\hline Characteristic & $\mathrm{N}=30$ \\
\hline Sex & Male: $17(56.7 \%)$ \\
Gestational age at birth & Median: 33.1 weeks \\
Gestational age at discharge & Median: 36.4 weeks \\
Gestational age at end of NG feeding & Median: 37 weeks \\
& Median: 8 days \\
No. of days NG fed at home & Range: $2-24$ days \\
Birth weight & Median: $1.94 \mathrm{~kg}$ \\
Discharge weight & Median: $2.18 \mathrm{~kg}$ \\
Weight at end of NG feeding & Median: $2.22 \mathrm{~kg}$ \\
Type of feeding & Breast: $18(60 \%)$ \\
\hline
\end{tabular}

